\title{
ANALYSIS, DESIGN AND IMPLEMENTATION OF A VACCINATION INFORMATION SYSTEM
}

\author{
Blirona Keraj ${ }^{1}$ and Igli Hakrama ${ }^{2}$ \\ ${ }^{I}$ Data Science \& AI, Saarland University, Saarbrücken, Germany \\ ${ }^{2}$ Computer Engineering, Epoka University, Tirana, Albania
}

\begin{abstract}
Nowadays, we cohabit with technology in an inseparable way. Acknowledging the importance of Immunization in population's health we emphasize the necessity of an intervention in the process of record-keeping and information flow that occurs during the vaccination and immunization procedures. This paper elaborates the process of analyzing, designing and implementing a Vaccination Information System e-Vacc using Unified Modelling Language
\end{abstract}

\section{KEYWORDS}

Vaccination Information System, UML, Data Processing, Vaccination Providers/Receivers, Design

\section{INTRODUCTION}

The way data is managed and handled is one of the main indicators of the efficiency and accuracy of the activity related to that data. Vaccination efficiency is a crucial metric that measures the quality of the national health care system. We are currently witnessing the collapse that can cause a contagious disease proving the importance and seriousness of this delicate topic. Undoubtedly, a qualitative and accurate information and historical data derived from vaccination is the best coach for future steps toward vaccination improvement.

Vaccination in Albania needs and deserves more than being handled manually and on paper registers and archives. The digitalization of this process will bring closer every party involved in vaccination, intensifying the cooperation for a better immunization procedure. To serve this need, a good solution would be a vaccination information system. This is an outcome of an iterative process from the analysis and design to the implementation of the system. Some of the steps are briefly presented in this paper using UML diagrams.

\section{BACKGROUND}

The interrelation between informatics and immunization is described by the transition from paper based to electronic health record. The systems already implemented in other countries and important indicators are put forward to support the idea of implementing a vaccination management system.

\subsection{Transition from Paper Based to Electronic Health Record}

An electronic health record is a repository of electronically maintained information about an individual's health status and health care, stored such that it can serve the multiple legitimate uses and users of the record. (Clement J. McDonald, 2014) Paper based record is a repository of individual's health information but stored in a media that constraints its functionality, paper.

EHR is flexible and takes the form user requires it, in contrary paper-based record which remains in the form it was stored, requires manual, time consuming manipulation with a high probability of errors. Health care and particularly vaccination is an information-intensive field. Vaccination providers are constantly collecting, gathering, reviewing, analyzing, communication information from many sources to make decisions. Information software related to health care is intended to facilitate the use of this information in different stages 
of delivery process. (A.B. Wilcox 2013) Main functions of health care related information systems are; acquiring, storing, summarizing and displaying data, generating reminders or other forms of decision support, facilitating communication and information exchange, supporting educational research and public health initiatives

\subsection{Successful Implementations}

One of the first countries that invested on immunization's improvement by implementing an IIS is the US (Novick, Lloyd F. MD, MPH 2014). These IIS are created based on the CDC criteria and are subject to some predefined Functional Standards whose main goal is to implement the best practices of immunization, address changes in technology and prioritize the IIS goals (Centers for Disease Control, 2019). The absence of a unified system causes lack of synchronization and ones that are privately funded are vulnerable toward privacy leaks. The four main strategic priorities for IIS are: enhancing performance, promote adherence to IIS standards, sustain the IIS community and influence and monitor the health IT environment.

Another country with a consolidated immunization system is England. The vaccination procurement and data management procedures are handled by 2 separated systems; ImmFrom, which is a Public Health England website used to both collect data on vaccine uptake for the national immunization program and provide ordering facilities for vaccines. CHIS is build in 3 sections (NHS England, August 2015); child health information service system, a child health record department (CHRD), a parent held child health record (PCHR). In Europe, also, many countries use an IIS but there are a few cases that implement the vaccination reminders, which are crucial for preventing latencies and other related consequences.

\section{ANALYSIS AND DESIGN OF VACCINATION INFORMATION SYSTEM (E-VACC)}

The process of developing an application includes a series of steps such as analyzing, designing, implementing and testing the application. The most useful tool used throughout these steps is Unified Modeling Language. UML is a standard language for writing the software blueprints. The UML diagrams are used to visualize, specify, construct the components of a software (Pressman, 1987).

The development of the application includes a series of interleaved iterative processes such as requirement elicitation, analysis, validation which are part of the requirement engineering activity. Each step is visualized using UML, starting with behavioral diagrams to continue with interaction diagrams, followed by the design process of the system with the structural diagrams and so on. The rest of the paper describes briefly some of the processes and steps toward the final design and implementation of the system.

\subsection{Requirement Analysis}

Requirement analysis is the process of collecting services, operations, features of a system, which are generally defined after consultation with all system stakeholders. (Terolli,2013). A detailed analysis on the vaccination process was conducted in collaboration with immunization service providers to have a clear idea what is expected from the application. The system will provide for each user their accounts and their functionalities, and this way ensure the data flow and user data interaction. The account creation cannot be done directly by the user himself, but according to the privileges and dependencies that users have with each other, to ensure a data protection at some extent.

Four priority points of e-Vacc are:

- Generating geographical and provider-level childhood vaccination coverage estimates

- Vaccination documentation including information of person vaccinated and the details related to the vaccines injected; vaccine type, lot number, manufacturer; injection site; dosage; and name, of person who injects the vaccine.

- Client reminder and feedback for provider/patient

- The inventory data registration, record of every order and automatic update calculations of available doses

- The patient side of the system including every detailed information for immunization processes and historical data 
Use case diagrams are used to illustrate the interactions user has with the system and the user's perspective of the application. These diagrams include main functionalities system has for each actor.

The system can be used by 8 users:

Admin: the main system manager. The admin's account credentials are provided by the systems' creators. The account creation of the vaccination providers will be done by registration requests. Admin will receive and decide upon the registration requests from Main Depot Managers and Local Admins.

Local Admin: shall manage every user employee categorized by position on the Health Center he has been assigned. Local admin zones to patronage nurses, who gain access to all the children's personal cards and vaccination schedules living inside that zone of the geographical area covered by the Health Center.

Maternity nurse: registers the child into the system through the New Born section, pass the security step, fill the form with personal and the maternity information. Proceeds with child's account creation specifying the credentials which will be given to child's parents. The last step is the personal card creation based on baby's personal number, Health Center and the zone.

Vaccinator Nurse: records every vaccination and this vaccination entry will be automatically entered in the Main Register of Vaccination and in the individual's personal card. Besides the vaccination entry, in case of any incident or unusual situation the vaccinator shall document it on the Special Cases section for later reference and historical data maintenance. The nurse must record the used and wasted items to update the local depot inventory. Vaccinator shall generate the quarterly coverage report.

Patronage Nurse shall access every patients' personal immunization schedule and health card, can view the Main Register of the zone assigned to. The patronage Nurse shall view the upcoming vaccination in weekly and monthly plan and must trigger the function that automatically calculates and sends the email reminders for upcoming vaccination 3 days in advance to every vaccination receiver.

Local Depot Manager: shall send vaccine orders each trimester to the Main Depot. Maintain orders from Health Centers of the district he covers and subtract the distributed doses. Specify the received order date so the system does the addition of the new doses and subtracts these amounts of doses from the inventory of the supplier Main Depot. Generate the Vaccine Demand Trimester Plans.

Main Depot Manager: shall view the list of all Local Depots and their managers, also, manage the orders requested by the local depots and also request doses from the main suppliers. Generate the Vaccine Demand Trimester Plans. Maintain inventory by checking the low doses warnings sent by the system.

Child(parent): is the child account used by the parent to follow his child's immunization. Each of them can view their vaccination history and track it based on the official vaccination schedule. Changes can be made at the profile section and a feedback form is provided. Three days before the vaccination appointment every individual is notified through email reminder.

\subsection{System Design}

System design is related to constructing the system architecture and also defines the system abstractions and their relationships. Realization of software design based on a set of programs represents the implementation of the system (Sommerville 2011). After an intensive requirement analysis the main modules of the e-Vacc Vaccination System are identified; Depots and vaccine inventory, Child's account and Vaccination data, Health Centers and vaccination providers organization.

Activity diagram provides a graphical representation of the flow of actions and their interaction. In the activity diagram is represented the process of child's registration in the system, the personal card initialization and the child account access. This process is done by the maternity nurse in every maternity in Albania. 


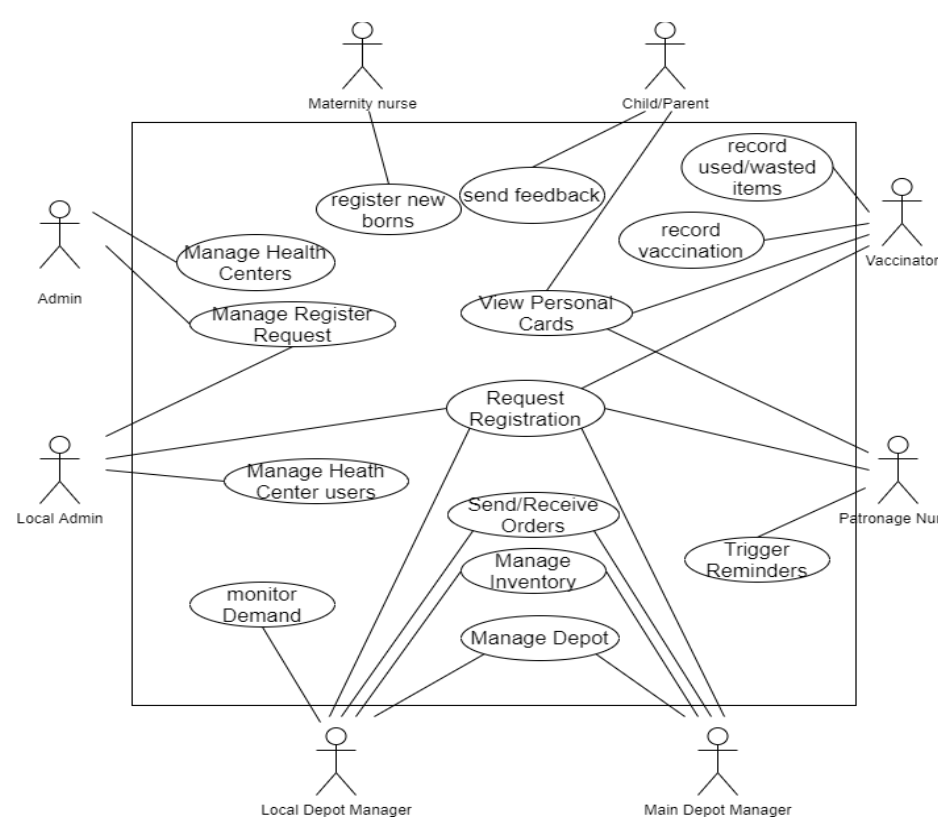

Figure 1. e-Vacc Use Case

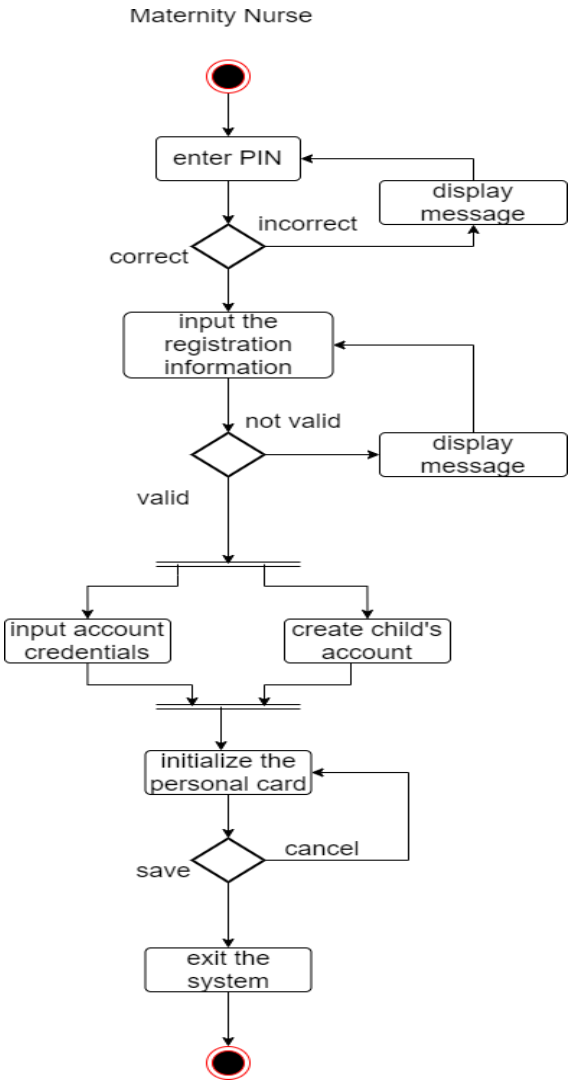

Figure 2. Child Registration Activity Diagram

Sequence diagrams are part of behavioral diagrams with the aim to represent the transition from one object to another as a function of time caused by different event. This sequence diagram is shown the vaccination entry record process done by the vaccinator for each child registered in a specific health centerThe personal Card of each child is generated by these vaccination entries. The main register, which holds the entries for every vaccination done, after each entry, fetches the data and gets updated.

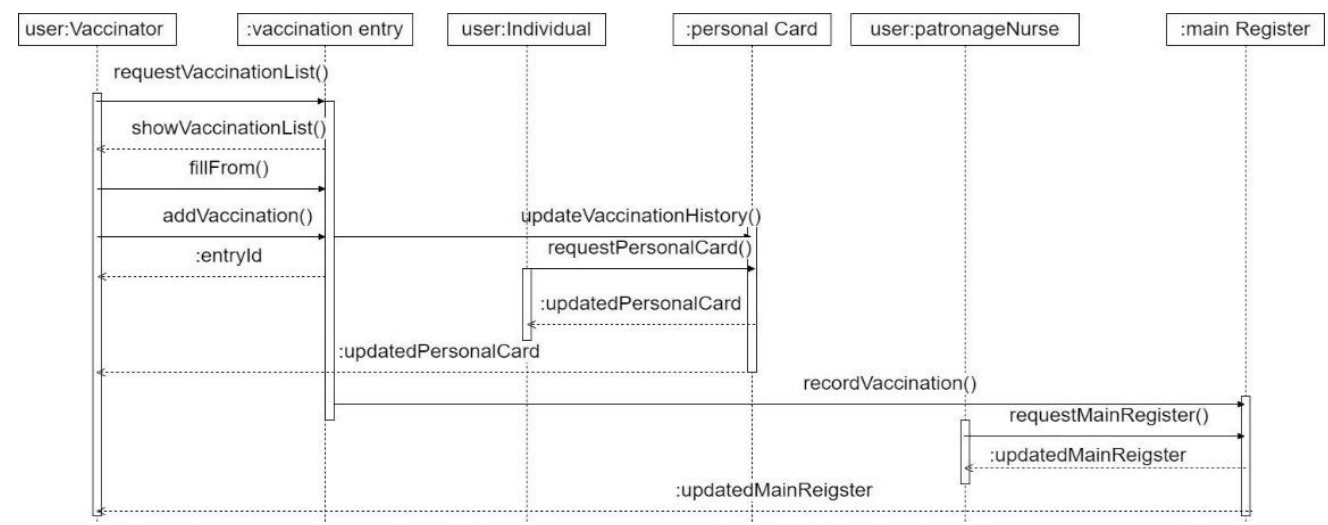

Figure 3. Vaccination entry Sequence Diagram 
The most important features of the system are the reminders. Everyday the system calculates the upcoming vaccination for the following 3 days, based on birthdays in the database. The patronage nurse triggers the reminder everyday just by pressing a button, and the child's parents receive the email reminders.

\section{IMPLEMENTATION}

The e-Vacc is developed as a web-application in order to be easily accessed requiring only a device with internet connection. This way not only vaccination providers but also individuals can access their vaccination data anytime they want. The technologies used for building this web-application are PHP, MySql, CSS, Bootstrap, HTML, Java Script, JQuery the interconnection of which manipulates data and offers a responsive and user-friendly web application. The process is ongoing and the system is tested only on experimental made-up data, because it is not yet implemented on Albanian Vaccination since it is a private initiative.

The system follows the actual Albanian Vaccination processes, despite some procedures that are not necessary when using e-Vacc. Such a case is the child registration which traditionally is done by the patronage nurse who has to deliver and receive the documents in person to and from the maternity. This task of registering the child in the system, opening a personal card and specifying the Health Center is done by the maternity nurse when the infant is born.

The implemented version of Vaccination Information System can be found on: http://stud-proj.epoka.edu.al/ evaccine/Vaccination\%20System/index.php

\section{CONCLUSION}

The success of immunization systems already implemented in different countries and the favorable infrastructure conditions in Albania push further the necessity of having a dedicated system for vaccination. The expected outcomes consist in reducing the vaccination providers workload, overcoming the lack of labor force, provide data efficiency, prevent vaccination latency, raise health institutions flexibility toward unexpected situations, data accuracy and availability to every individual.

Undoubtedly, aforementioned features require to be expanded adding more functionalities and modules to achieve a fully developed Immunization System. One of them is the module of health records from routine check-ups, by the Family's Doctor, of children from 0 to 6 years old. This will be the right complementary part for this application to settle a fully functioning and incorporative Immunization System.

\section{REFERENCES}

Centers for Disease Control and Prevention. (2019, October 7). Basics of Immunization Informatoin Systems. Retrieved from https://www.cdc.gov/vaccines/programs/iis/about.html

Clement J. McDonald, P. C. (2014). Biomedical Informatics (Vol. Electronic Health Record Systems). London: Springer . doi:https://doi.org/10.1007/978-1-4471-4474-8_12

NHS England. (2015, August 10). Child Health Information Services (CHIS)Provider Service Specifications. Retrieved from https://www.england.nhs.uk/commissioning/wpcontent/uploads/sites/12/2013/05/chis-provider-service-spec.pdf

Novick, L. F. (2014, June 6). Immunization Information Systems. Journal of Public Health Management and Practice . doi:10.1097/PHH.0000000000000111

Pressman, R. S. (1987). Software Engineering: A practitioner's approach. New York: McGraw-Hill. Retrieved from https://dinus.ac.id/repository/docs/ajar/Pressman-Software_Engineering-9ed.pdf

Somerville, I. (2011). Software Engineering (9th ed.). Addison-Wesley.

Terolli, E. \& Hakrama, I., 2013, Modeling a Career Office Information System with UML, 2nd International Symposium on Computing in Informatics and Mathematics, Tirana, Albania, pp. 98-108. 